\title{
ANALYSIS OF ANXIETY AND IMMERSIVE PROCESS IN DIGITAL GAMES USING HTC VIVE
}

\author{
Gabriel Batista Caldeira and Rosilane Ribeiro da Mota \\ Hochschule Furtwangen University, Germany \\ Pontifícia Universidade Católica de Minas Gerais, Brazil
}

\begin{abstract}
This work aimed to evaluate the anxiety level and the level of immersion of a player when using the virtual reality HTC Vive to interact with a digital game. With this objective in mind, a digital game was developed that is of runner genre. Assessing the level of anxiety was carried out using the SUDS and IDATE questionnaires. The immersion evaluation process was conducted through the IPQ questionnaire. Two contexts are proposed to assess user anxiety and the immersive process. In the first, the player interacted with the game using the keyboard/mouse, answering the questionnaires at the end. In the second context, the same player interacted with the game through HTC Vive, also answering questionnaires. It was observed that the level of anxiety of the participants did not increase significantly and that the level of immersion had a significant increase, demonstrating that it is justified to use the HTC Vive to obtain a better immersive process. This was also the first study to indicate that games can have a positive influence in times of great anxiety and stress, such as exams for university students.
\end{abstract}

\section{KEYWORDS}

Virtual Reality, HTC Vive, Immersive Process, Anxiety

\section{INTRODUCTION}

Interaction with digital games is a practice that is increasing in popularity, in the Entertainment Software Association (2015) it is reported that $72 \%$ of the general population and $97 \%$ of adolescents, interact with digital games regularly (McMahan, Parberry, Parsons 2015). For this reason, it is necessary to carry out the analysis of the productive process of games and it is also important to study the benefits that digital games can bring, as Granic, Lobel e Engels (2014) mention in their work studies on the cognitive aspect in this area are very scarce and so there is a need for deeper analyzes on these processes.

In the study carried out by Granic, Lobel e Engels (2014), it is stated that it is in the theory of uses of gratuities which lies one of the main reasons why individuals use media: manage the state of their mood and emotional state. With that, it is possible to affirm that digital games are among the most effective and efficient way to generate positive feelings in children and young people. Some studies claim that there is a relationship between interacting with digital games and improving or increasing the flow of positive emotion (Russoniello, O'Brien and Parks 2009)(Ryan, Rigby and Prybylski 2006). Studies suggest that puzzle games, short-term commitments games and games that have a high degree of accessibility (e.g. Angry Birds, Bejewled II) - can improve the mood of the players, promoting relaxation and decreasing anxiety (Russoniello, O'Brien and Parks 2009).

Anxiety consists of an unpleasant emotion that can be divided into state and trace (Spielberger, Gorsuch, Lushene 1970). Anxiety-state is different in intensity according to time, that is, it refers to acute situations that correspond to momentary episodes. Trait anxiety refers to the differences that each individual presents for behavioral responses (Spielberger, Gorsuch, Lushene 1970). This makes it possible to assess the state of anxiety in a person in different situations, such as for example after a person interacted with a digital game.

In the study by Alsina-Jurnet and Gutiérrez-Maldonado (2010), it can be seen one of the main characteristics in the treatment of psychological disorders through the use of virtual reality is the sensation of presence or feeling of being present. One of the most important aspects of the illusion is that the virtual environment evokes approximately the reactions and emotions as an experience in the physical world. This means that the sense of presence is essential and can be used as a metric to assess the player immersion. We are using the term immersive process as the sense of presence in this paper. 
With the topics presented previously, the general objective of this work was the evaluation of a player's anxiety and immersive process when he/she is using a virtual reality device. For this purpose, a game was developed that was used for the evaluation of anxiety and immersion when a player was using virtual reality glasses, HTC Vive.

\section{RELATED WORKS}

In Alsina-Jurnet and Gutiérrez-Maldonado (2010) a study was carried out in which five user characteristics were evaluated: anxiety, spatial intelligence, verbal intelligence, personality and experience with computers, all of which could influence the feeling of being present. This was the first study that researched the relationship between spatial intelligence with the feeling of being present in a virtual environment. In this work, it was identified that the greater the user's anxiety the greater it was his feeling of being present.

In the work of Perandré and Haydu (2018), an analytical-behavioral therapeutic intervention was performed for participants with social anxiety disorder. For this, virtual reality was used as a therapeutic resource. This job concluded that intervention with virtual reality caused a therapeutic effect and better confrontation in the face of social interactions.

In the work of Granic, Lobel and Engels (2014), a research was made on the effects of positive aspects of interacting with digital games, focusing on four main domains: cognitive, motivational, emotional and social. Considering these potential benefits is important, in part, because the nature of these games has changed drastically over the past decade, becoming increasingly complex, diverse, realistic and social in nature. The goal of this work was to provide sufficiently strong evidence and theoretical reasoning to inspire new education programs and research on mental health benefits, little explored in digital games.

In Alsina-Jurnet and Gutiérrez-Maldonado (2010), we could see a research aiming to understand personal characteristics and the feeling of being present in a virtual environment. The study of Perandré and Haydu, (2018) that uses virtual reality as a therapeutic resource. The research of Granic, Lobel and Engels, (2014), shows how games are dramatically changing. But in none of these researches it is studied how virtual reality interferes with the immersion and anxiety of a player. That is why the study proposed by this paper is important.

\section{METHODOLOGY}

In this section, the development of the digital game will be addressed, the questionnaires used to assess anxiety and immersion, carrying out the related experiments.

\subsection{Hardware}

For processing the digital game with suitability for virtual reality a notebook was used with the brand Asus and ROG Strix GL702ZC model. This notebook has the Windows 10 operating system, 64bit, 8GB RAM, 1TB Hard Disk, AMD Ryzen 5 1600, 3.2 GHz processor 6 core, 12 threads and an AMD Radeon graphics card RX 580, (Laptop) 4096MG GDDR5 1077Mhz core clock. In the experiments, the user used the notebook and played using keyboard and mouse, viewing the game directly on the notebook screen. As for playing in virtual reality, the players used HTC Vive connected directly to the notebook.

\subsection{Questionnaires}

Two questionnaires were used to carry out the evaluation of anxiety, the SUDS (subjective units of discomfort scale), which provides a subjective anxiety scale and the STAI (strait-trait anxiety inventory), which provides trait and state of anxiety. The immersive process was evaluated through the IPQ questionnaire (Igroup presence questionnaire).

The IPQ questionnaire (Igroup presence questionnaire) is a self-report type of questionnaire, it was initially 
created by Schubert, Friedmann and Regenbrecht (2001). In its first version, as well as in the Portuguese version used in this work, it was intended to measure the following variables: spatial presence, involvement, experienced realism and global presence. To measure these variables, there are 14 questions proposed in a five-point Likert scale format, using different anchors. In this work, the Portuguese version of the questionnaire IPQ was used. This version was translated and validated by Vasconcelos-Raposo et al, (2016).

The spatial presence variable refers to the feeling of being physically inserted in the virtual environment, since the involvement refers to the attention that the person gives to the virtual environment and the involvement experienced by it. Another aspect is the experienced realism it represents the subjective experience of realism in the environment virtually. The global presence variable is the feeling of being present in the virtual environment (Vasconcelos-Raposo et al, 2016).

In the SUDS questionnaire (Subjective Units of Discomfort scale), participants indicate the level of their perceived anxiety on a scale ranging from 0 (not at all anxious) to 10 (very anxious) (Wolpe, 1969). With this questionnaire it is possible to obtain the subjective level of anxiety of an individual, and this test was used in conjunction with the STAI test to assess the anxiety of the players. To assess the anxiety obtained by this questionnaire, a comparison was made of the values obtained after the interaction with keyboard/mouse with those obtained after interacting with HTC Vive.

The STAI questionnaire (Strait-trait Anxiety Inventory) is of the self-report type, evaluating the state and a person's anxiety trait. For this work, only the anxiety state scale was used due to established objectives. The questionnaire consists of 20 items that are scored in an interval of 1 (not significant) to 4 (very significant). The Portuguese version of the Strait-trait anxiety inventory (STAI) was used, called Inventário de ansiedade traço-estado (IDATE)(Spielberger, Gorsuch and Lushene, 1970),(Biaggio, 1990).

Each question has a corresponding score according to the answer of the player. However, for questions with a positive type, the score is reversed (for example, if the patient responded 4 for these questions, an inverted score is given, a value is assigned 1 in coding) (Biaggio, 1990). The assessment is made by adding all

the scores found in the questionnaire, respecting the rule for positive and negative issues. This value, therefore, varies between 20 to 80 and the higher the value, the greater will be considered the person's anxiety.

\subsection{Game Development}

In order to explore the level of anxiety of a player, a controlled environment was needed. A digital game was created with this goal in mind. For game development the Unreal Engine 4 (UE4) and the Blueprint programming language were used. Among the reference games, at the time of the experiments, there was none that met the requirements among them that presented the same gameplay for PC and VR. Thus it was decided to develop a simpler game, placing the gameplay on both platforms as constant to assess the difference in anxiety and immersion in the two platforms.

It was decided to use the Unreal Engine for the development of the game, because it generates final results with more professional quality (Dickson, Block and Echevarria, 2017).

A digital game of the runner genre was developed, consisting of a race where the player controls a character and must dodge various obstacles. To control the character by dodging obstacles and to move around in the virtual world, the player uses the following commands: forward, back, right, left and jump. If the player is hit by an obstacle, the map is restarted and the player must restart the course.

For a better experience of the player this game has three levels of difficulty. These difficulties were designed so that both experienced players and inexperienced ones could be challenged. Through the initial menu the player can choose the desired difficulty, and this difficulty can also be changed during the game via a menu break.

The game has 4 stages, each of which has obstacles with different elements. In the first stage, the obstacles are composed of spheres and rivers of lava, representing the element of fire. In the second stage, obstacles are composed of spheres and rivers of water. In the third stage, there are two types of obstacles, spheres of smoke and blue rays, and at this stage the player also walks over white smoke, and each of those elements represents air. In the last stage, the obstacles are blocks of stone that represent the element from the earth. These elements were designed to characterize a better narrative related to the game scenario.

When the user finishes the game, a victory screen appears for the player, in which the player could choose between restarting the game or quitting. 
Figures 1 and 2 show the first and last stage of the digital game developed. In the left part of these figures it is possible to see the initial phase of the game in which the player has to avoid some lava objects and on the right you can see the final part where the participant has to dodge some earth cubes.

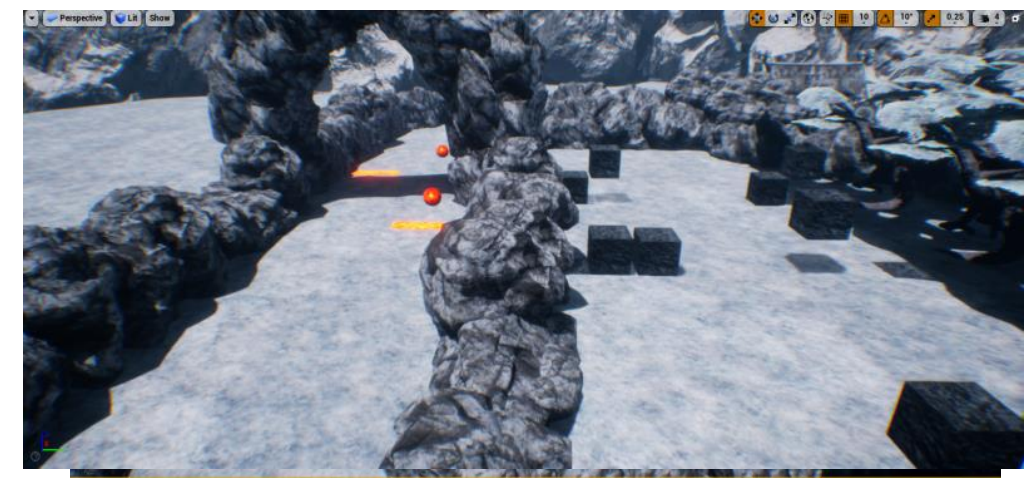

Figure 1. The first stage of the game on the left and last stage on the right. Source: Designed by the author

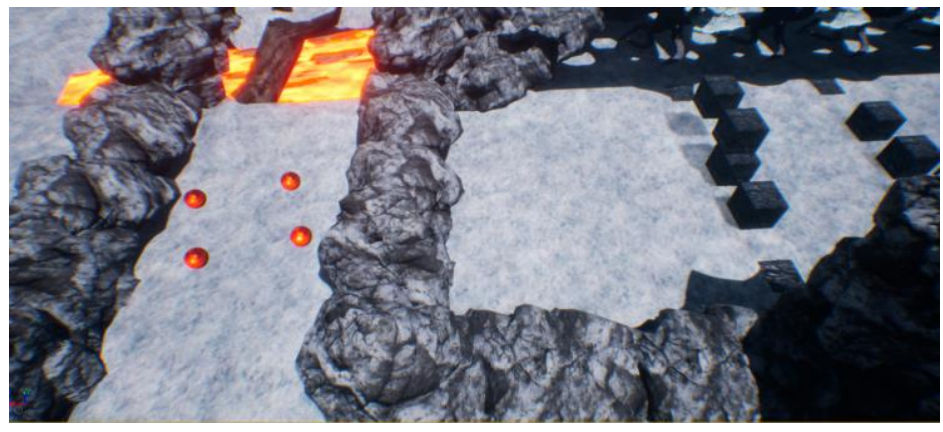

Figure 2. The first stage of the game on the left and last stage on the right. Source: Designed by the author

The corridor to the left of Figure 1 provides access to the corridor in the left part of Figure 2, this part being considered the first part of the game. That part has 4 obstacles: the first deals with an immobile sphere with a part of lava down; the second obstacle is a sphere that keeps moving along the corridor and it also has a part of lava under it; the third is 4 spheres that rotate in counterclockwise direction, and the fourth is a tree trunk that needs to be crossed.

It is possible to see the second stage of the game in Figure 3, and the third stage was indicated in Figure 4. For the composition of the scenario, there is a village in the second stage and some trees and, as obstacles, there are spheres of water and the player must avoid falling into the river. In the third stage, the player walks through a region with white smoke and must dodge from rays and blue spheres.

\subsection{Design of Experiments}

Based on the work done by Alsina-Jurnet and Gutiérrez-Maldonado (2010), a study was proposed that assessed a player's anxiety when interacting with a digital game using HTC Vive.

An experiment was carried out with thirty university students. The students belonged to a private institution, 24 of them male and 6 female. It was defined that the participants would meet the same criteria, namely: they would not be under the effect of any form of controlled medication; they did not have any psychological disorder; they were not receiving any form of psychological therapy; did not have any kind of medical problems, like heart disease or epilepsy; they had Portuguese as their native language.

These criteria are important because if any of the players suffer from any psychological disorder or if they are receiving any type of therapy, these people could influence the result of the level of anxiety and the data would not be reliable. 


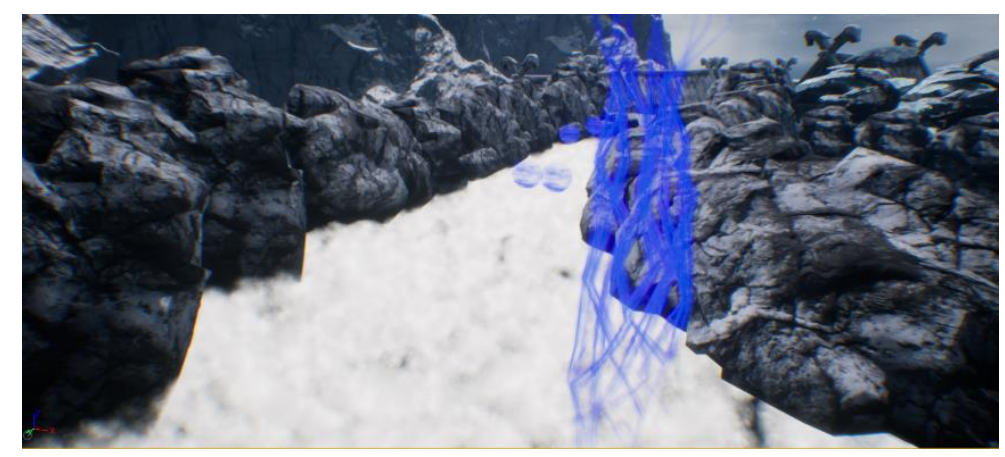

Figure 3. The second stage of the game. Source: Designed by the author

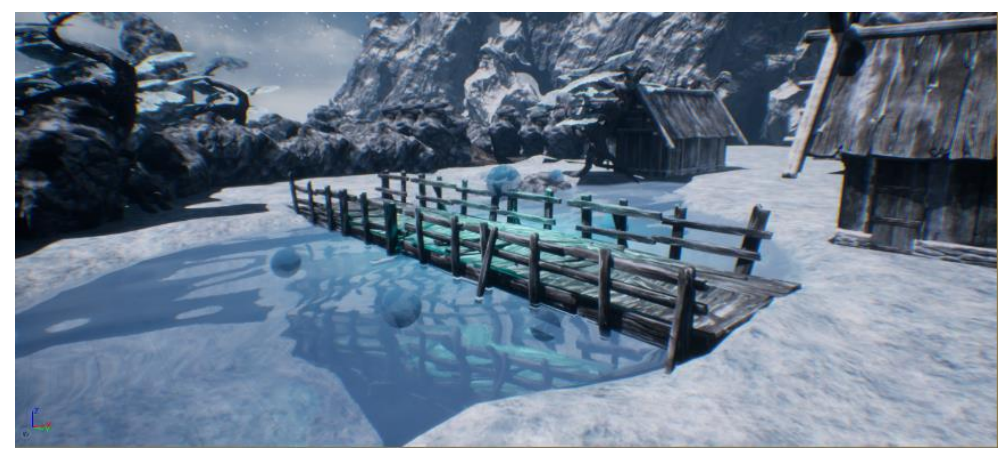

Figure 4. The third stage of the game. Source: Designed by the author

At the beginning of the tests it was presented to the participants how the whole process would work, and if the participants had any questions. Those would be clarified. Soon after, the participants filled in their personal information in a questionnaire that was available online on Google Forms.

The experiment had an average duration of 20 minutes for each participant, of which, 6 minutes were to interact with the game via HTC Vive device, 6 minutes to play via keyboard/mouse and 8 minutes to filling in the questionnaires. Interaction with the game via keyboard/mouse or with virtual reality glasses was defined by the time of six minutes or if the user could complete the game. In other words, the game was finished and the players answered the questionnaire, if the player played for 6 minutes without success in finishing it or if he managed to finish the game before the time. The players were not aware of the 6 minutes time and were just playing. This kind of measure was taken to avoid affecting the level of anxiety of the players.

The experiment was divided into 2 stages. In the first stage the players interacted with the game through keyboard/mouse, answering the SUDS, IDATE and IPQ questionnaires when finished. The data obtained in that first stage were defined as reference data for the comparison with the data that were obtained in the second stage. For the second stage the players used HTC Vive for interacting with the digital game, responding to the same questionnaires once completed. The answer to the questionnaires was available online and if the participants desired, a copy was sent by email to the participant.

With the completion of the experiments, then the data from the experiment were analyzed. The change in anxiety was assessed through the questionnaires SUDS and IDATE. The change in the immersive process was accessed through the IPQ questionnaire. An analysis was made of the score obtained in the first and second part of the experiments to identify the change in anxiety and immersive process obtained with the use of HTC Vive. 


\section{RESULTS}

Due to the fact that the game was proposed and developed by the researcher, an initial test was performed with a participant to find out if any game modification were necessary. This test was performed in the same way as the other tests, except for the time, which in this case lasted 40 minutes. With the completion of this test, it was identified that the difficulties of the game were in levels much higher than expected. With that in mind, obstacle speeds have been reduced to facilitate dodging of the same by the players.

Another problem was identified in this pilot test, it was related to one of the particle systems used that caused a malfunction in the game. This particle system was located in the first stage of the game and simulated fire. The problem was that this system caused an FPS drop in this area. An error was also identified that did not allow the menus to be displayed for HTC Vive. The correction of this error was made by assigning hotkeys to different difficulties of the game, making it possible to activate different levels without menu access.

In the end, the results of this Alpha test were not considered with the values of the general tests, as they are dealt with different experiences for the player.

Approximately $33.3 \%$ of participants completed the game via keyboard/mouse. With HTC Vive, only $13.3 \%$ of the participants managed to complete the game. It was reported by users a greater difficulty in interacting with the game in virtual reality.

With the SUDS questionnaire it was possible to identify that if you play with HTC Vive the subjective anxiety level of a player had an average increase of $10.3 \%$. This was the first questionnaire for the evaluation. With the values that are shown in Table 1 for the SUDS questionnaire, it is possible to verify that users reported having a little greater anxiety when playing with the virtual reality glasses. This can be a result of strangeness in the first use of the device for most of the participants.

As seen in Spielberger, Gorsuch and Lushene (1970) and Biaggio (1990), the questionnaire score STAI consists of a value from 20 to 80 . On this scale, there is no definite cut due to the fact that the level of anxiety may vary according to the individual characteristics and sampling. This score is a summation with all the questions present in the questionnaire taking into account that the negative and positive questions have different values in the score. In the work of Biaggio (1990) it is possible to see that in neutral conditions the average for the STAI-state is 39.63. This value will be considered as a reference for this work. The values obtained through the IDATE are shown in Table 1.

With the results of the IDATE-state questionnaire, it is possible to see that the average increase in the anxiety of players when using HTC Vive was 2.6 points. The increase in anxiety was not statistically significant, if compared to the total of 80 questionnaire points or taking note that each question is worth 4 points. However, about 9 people experienced a significant increase in anxiety, this may be due to the fact that the HTC Vive is relatively new and it might be of difficult access for the general public. This significant increase should be considered if any work is being done with the user, as in Perandré and Haydu (2018), as there is a possibility that the user feels greater anxiety for interacting with the virtual reality glasses and this must be considered.

It was observed that about $10 \%$ of the population presented dizziness when using HTC Vive. For this reason, these people's anxiety scores were higher than normal. The relative increase in anxiety was about 3 points in SUDS and 8 points in the IDATE questionnaire. When participants reported dizziness, the test was stopped until these participants felt better, and then the questionnaires were applied. It was reported by participants that this dizziness was only momentary and did not present any major risk. It was mentioned by participants who suffered this type of adversity, they were extremely tired, and the tiredness may have contributed to the feeling of dizziness with the use of virtual reality glasses. To do this type of connection of tiredness and dizziness presented with reality glasses, a new study is needed that seeks to understand the correlation between the two.

The evaluation of the immersive process was carried out through variables presented in the IPQ questionnaire that are shown in Table 1. With the results obtained through this instrument, it was possible to see that all variables portrayed higher values for using HTC Vive regarding keyboard/mouse use. In that way, it can be inferred that the device confers a higher level immersion for players. 
Table 1. Data of the questionnaires SUDS, IDATE and IPQ

\begin{tabular}{lll}
\hline SUDS & IDATE & IPQ \\
\hline Average mouse/keyboard: 4.63 (SD: & Average mouse/ keyboard: 37.46 (SD: & Increased in spatial presence: 0.58 \\
$2.14)$ & $7.25)$ & \\
HTC Vive average: 5.66 (SD: 2.78) & HTC Vive average: 40.06 (SD: 9.40) & Increase in involvement: 0.635 \\
17 people had an increase & 13 people had an increase & Increased in realism experienced: 0.55 \\
6 people had a decrease & 14 people had a decrease & Increased in global presence: 0.59 \\
\hline
\end{tabular}

As seen in Granic, Lobel and Engels (2014), games can promote relaxation and decreased anxiety. In this work it was possible to confirm this statement due to the fact that the anxiety levels found by the IDATE questionnaires and SUDS are below average for the keyboard/mouse interaction and slightly above average for the interaction with the HTC Vive. With the increase of immersion, it is possible then that the players experience these positive effects that games bring in a more intense way. As the player has a greater feeling of being present in the virtual environment due to the HTC Vive, any kind of experience he has will be amplified.

The average anxiety in the IDATE-state for students in the exam period of the subjects was 45.71, as seen in Biaggio (2010). In this work, the average was 37.46 using keyboard/mouse. And considering that the tests of this work were applied during a final exam period of university students, which is a period that students show a significant increase in the state of anxiety and stress, the result of this experiment can be indicative that the game developed for this work can have offered a slight reduction in the state of anxiety of students. Even with the highest score obtained by the IDATE-state of 40.06 by the participants playing with virtual reality glasses, there was a decrease. If the value when the game was played via a keyboard/mouse 37,46, this decrease further reinforce the previous statement.

\section{CONCLUSION}

The objective of this work was to evaluate anxiety and the immersive process for the HTC Vive using a developed game for this research of the runner genre. This work was based on the research of Alsina-Jurnet and Gutiérrez-Maldonado (2010). The choice of the runner genre indicated the necessity of new evaluations, by the observation that this genre may not be adequate for VR. In the future new researches will be made involving other genres, with that we are going to be able to understand the difference between levels of anxiety and immersion for games VR and non VR in different games genres. In the developed game for this research $10 \%$ of the total players had motion sickness while playing in VR. In future works new elements in the interface and gameplay could be tested to reduce this sensation.

As was discussed in the results subsection, through the application of the SUDS and IDATE questionnaire, there was no significant increase in the average anxiety of users. However, some users have experienced a significant increase in anxiety and in these specific cases, it is suggested that HTC Vive should be used more carefully. At the work of Perandré and Haydu (2018), virtual reality was used as a therapeutic resource, but it was not considered that users could have greater anxiety when wearing glasses of virtual reality. Due to these factors, it is advisable that introductory exercises are done with the devices so that these users will have a more pleasant experience.

This work indicated the positive aspects that games can promote during periods of stress and anxiety, for example, during exams for college students. As previously seen, there is a factor that may indicate that the game has reduced students anxiety while they were playing. It is proposed as a future work a study that analyzes this indication and can confirm or refute the findings found in this work. It would also be interesting to develop another line of research aimed at studying the development of games to decrease anxiety in individuals who are in a state of extreme anxiety. It could also be proposed the same experiment encompassing more questionnaires and devices. Another future research that could be proposed is to use a commercial VR game and do the same measurements proposed in this research, after that we can compare with the results found in this work using the VR game developed by the researcher. 


\section{REFERENCES}

A. Biaggio, 1990. A decade of research on state-trait in brazil. in Cross-cultural anxiety, C. Spielberger and R. Diaz-Guerrero, Eds. New York: Taylor Francis, pp. 157-167.

C. Russoniello, K. O'Brien, and J. M Parks, 2009. Eeg, hrv and psychological correlates while playing bejeweled ii: A randomized controlled study. vol. 144, pp. 189-92, 022009.

C. Spielberger, R. Gorsuch, and R. Lushene, 1970. Manual for the state-trait anxiety inventory. Consulting Psychologists Press.

Entertainment Software Association, 2015. Essential facts about the computer and video game industry: Sales, demographic, and usage data. Available at: <http://www.theesa.com/wp-content/uploads/2015/04/ESAEssentialFacts-2015.pdf>. Accessed: 30 May 2017.

I. Alsina-Jurnet and J. Gutiérrez-Maldonado, 2010. Influence of personality and individual abilities on the sense of presence experienced in anxiety triggering virtual environments. International Journal of Human-Computer Studies, vol. 68, no. 10, pp. 788 - 801. [Online] Available: https://doi.org/10.1016/j.ijhcs.2010.07.001

I. Granic, A. Lobel, and R. C. M. E. Engels, 2014. The benefits of playing video games. American Psychologist, vol. 69, no. 1, pp. 66-78. [Online]. Available at: doi:10.1037/a0034857

J. Vasconcelos-Raposo, M. Bessa, M. Melo, L. Barbosa, R. Rodrigues, C. M. Teixeira, L. Cabral, A. A. Sousa, 2016. Adaptation and validation of the igroup presence questionnaire (ipq) in a portuguese sample. Presence, vol. 25, no. 3, pp. 191-203.

J. Wolpe, 1969. The practice of behavior therapy. New York, NY: Pergamon Press.

P. E. Dickson, J. E. Block, G. N. Echevarria, and K. C. Keenan, 2017. An experience-based comparison of unity and unreal for a stand-alone $3 d$ game development course. pp. 70-75, 2017. [Online] Available at: http://doi.acm.org/10.1145/3059009.3059013

R. M. Ryan, C. S. Rigby, and A. Przybylski, 2006. The motivational pull of video games: A self-determination theory approach. American Psychologist, vol. 30, no. 4, pp. 347-363. [Online] Available at: doi:10.1007/s11031-006-9051-8

T. McMahan, I. Parberry, and T. D. Parsons, 2015. Modality specific assessment of video game player's experience using the emotiv, Entertainment Computing. vol. 7, pp. $1-6,2015$. [Online] Available at: https://doi.org/10.1016/j.entcom.2015.03.001

T. Schubert, F. Friedmann, and H. Regenbrecht, 2001. The experience of presence: Factor analytic insights. Presence: Teleoperators and Virtual Environments, vol. 10, no. 3, pp. 266-281. [Online] Available at: https://doi.org/10.1162/105474601300343603

Y. H. T. Perandré and V. B. Haydu, 2018. Um programa de intervenção para transtorno de ansiedade social com o uso da realidade virtual, Temas em Psicologia, vol. 26, pp. 851 - 866, 06. 